\title{
Epidural abscess and spondylitis caused by Veillonella parvula in a man on hemodialysis
}

\author{
Masaru Kurihara ${ }^{1}$, Itaru Tamaki ${ }^{1}$, and Yasuharu Tokuda ${ }^{2}$ \\ ${ }^{1}$ Urasoe General Hospital \\ ${ }^{2}$ Muribushi Okinawa Teaching Hospitals
}

June 4, 2021

\begin{abstract}
Clinical Case Report

Running title: Epidural abscess and spondylitis caused by

Veillonella parvula

Masaru Kurihara, Itaru Tamaki, Yasuharu Tokuda

[Institutions]

Correspondence: Masaru Kurihara

Department of Hospital Medicine, Urasoe General Hospital

Okinawa, Japan

Tel: $+81-98-878-0231$

Fax: +81-98-878-0231

E-mail: masarukurihara1025@gmail.com
\end{abstract}

Veillonella species rarely cause serious infections, but the incidence of infection has been increasing among immunocompromised individuals. This case of an epidural abscess and spondylitis caused by Veillonella parvula in a hemodialysis patient illustrates the importance of performing anaerobic blood culture in immunocompromised patients with signs of infection.

Epidural abscess and spondylitis caused by Veillonella parvula in a man on hemodialysis

\section{Key Clinical Message}

Veillonella species rarely cause serious infections, but the incidence of infection has been increasing among immunocompromised individuals. This case of an epidural abscess and spondylitis caused by Veillonella parvula in a hemodialysis patient illustrates the importance of performing anaerobic blood culture in immunocompromised patients with signs of infection.

Keywords: Infectious diseases, Opportunistic infections, Veillonella infections, Gram-negative bacteria, epidural abscess, hemodialysis

\section{Introduction}

Veillonella is an anaerobic gram-negative coccus that inhabits the oral cavity, gastrointestinal tract, and female genital tract. ${ }^{1-3}$ Thirteen species of the genus have been identified, of which six species have been 
isolated from the human oral cavity. ${ }^{4}$ Veillonella species were formerly considered to be non-pathogenic and to rarely cause serious infections, but in recent years, there has been an increasing number of reports of human infections among immunocompromised individuals. ${ }^{5}$ Here, we report a rare case of an epidural abscess and spondylitis caused by Veillonella parvula .

\section{Case History}

A 52-year-old Japanese man presented to the emergency department of our hospital with a 1-week history of low back pain. He had also developed difficulty in walking over the previous 2 days. He had a 10-year history of chronic renal failure caused by glomerulonephritis and was on hemodialysis three times a week. He had smoked 20 cigarettes/day for 30 years and was an occasional drinker. On examination, he was alert, with a temperature of 37.0 , blood pressure of $105 / 74 \mathrm{mmHg}$, respiratory rate of 32 breaths/min, and heart rate of 180 beats/min. No abnormalities were detected on respiratory or cardiac examination. Abdominal examination showed tenderness over an area from the right buttock to the right thigh. There was no redness or tenderness at the dialysis shunt site, located on the left forearm. Laboratory test results on admission showed a white blood cell count of 16,200 cells $/ \mu \mathrm{L}$, hemoglobin level of $11.8 \mathrm{~g} / \mathrm{dL}$, and platelet count of $102,000 / \mu \mathrm{L}$. His serum potassium $(8.0 \mathrm{mmol} / \mathrm{L})$ and C-reactive protein $(41.7 \mathrm{mg} / \mathrm{dL})$ levels were elevated. His other blood test results are shown in Table 1.

\section{Differential diagnosis, investigations, and treatment}

He was admitted to the hospital and underwent emergency hemodialysis to correct the hyperkalemia, and his serum potassium level decreased to $4.5 \mathrm{mmol} / \mathrm{L}$. On day 2 of admission, he developed a fever $\left(40.1^{\circ} \mathrm{C}\right)$, and his right lower back pain worsened. Pelvic magnetic resonance imaging (MRI) revealed findings suggestive of an epidural abscess and spondylitis at the L5/S1 intervertebral disc (Figure 1). Blood culture revealed anaerobic gram-negative cocci. Based on these findings, the patient's condition was diagnosed as spondylitis and epidural abscess caused by anaerobic bacteria. Antimicrobial therapy was initiated with intravenous ampicillin/sulbactam (3 g 6-hourly). Testing using a VITEK-ANC Card@ identified the pathogen as a Veillonella species. and $16 \mathrm{~S}$ rRNA polymerase chain reaction identified the species as Veillonellaparvula . Based on the susceptibility by the micro liquid dilution method (Table 2), the antimicrobial agent was changed to ampicillin (2 g 6-hourly).

\section{Outcome and follow-up}

After initiation of ampicillin, the clinical and imaging signs of inflammation gradually improved. Antibiotics were administered for 58 days, and the patient was discharged on day 60 . He has been followed up further for 6 months since his discharge, and has not experienced a recurrence.

\section{Discussion}

This is a rare case of epidural abscess and spondylitis caused by V. parvula . Hirai et al. ${ }^{6}$ reviewed the literature from 1976 to October 2015 and found 31 cases of Veillonella infection in humans. Of these cases, five were musculoskeletal infections caused by $V$. parvula, including four spinal infections. There has been only one case of an epidural abscess caused by $V$. parvula reported previously. ${ }^{7}$ To our knowledge, the current case is the first report of human V. parvula infection in Japan. The previously reported case of an epidural abscess caused by $V$. parvula ${ }^{7}$ was in a cancer patient, and our patient was on hemodialysis; therefore, V. parvula appears to be an opportunistic pathogen that affects immunocompromised patients. Previous reports have indicated the importance of anaerobic culture for identifying this pathogen ${ }^{6}$ in our case, anaerobic culture isolated the organism, and further investigation revealed the species, suggesting that anaerobic culture should be considered in patients with signs of an epidural abscess, especially in immunocompromised patients.

Most Veillonella spinal infections reported to date have been associated with a subacute course of lower back pain from 1 week to 4 months. ${ }^{1,8,9}$ Spinal infections may develop serious complications if the diagnosis is delayed. ${ }^{10}$ Our case was diagnosed early using MRI. MRI should be considered in immunocompromised patients with signs of musculoskeletal infection with low back pain, such as our case. 
In conclusion, this is the first case of epidural abscess and spondylitis caused by $V$. parvula reported in Japan. Musculoskeletal infection caused by Veillonella can lead to chronic back pain in immunocompromised patients, and early diagnosis using MRI and anaerobic culture are recommended.

Author Contributions:

All authors critically revised the report, commented on drafts of the manuscript, and approved the final report.

Conflict of Interest: None declared.

Funding: This study was supported by a grant that was provided by the Japan Primary Care Association project for Developing Future Leaders in Research (grant number: 2019B002).

Consent for Publication: The patient has provided informed consent for publication.

\section{References}

1. Kishen, T. J., S. T. Lindstrom, G. Etherington, and A. D. Diwan. 2012. Veillonella spondylodiscitis in a healthy 76-year-old lady. Eur Spine J 21(Suppl 4):413-417.

2. Chen, Y. C., P. H. Ko, C. J. Yang, Y. C. Chen, C. J. Lay, C. C. Tsai, et al. 2016. Epidural abscess caused by Veillonella parvula : case report and review of the literature. J Microbiol Immunol Infect 49:804-808.

3. Baker, S., and R. Allyn. 2017. Lytic lesions: looking lethal but leaving room for a simple cure? A case of Veillonella spinal osteomyelitis. JMM Case Rep 4:e005108.

4. Mashima, I., and F. Nakazawa. 2015. Interaction betweenStreptococcus spp. and Veillonella tobetsuensis in the early stages of oral biofilm formation. J Bacteriol 197:2104-2111.

5. Gouze, H., L. Noussair, I. Padovano, E. Salomon, M. de Laroche, C. Duran, et al. 2019. Veillonella parvula spondylodiscitis. Med Mal Infect 49:54-58.

6. Hirai, J., Y. Yamagishi, T. Kinjo, M. Hagihara, D. Sakanashi, H. Suematsu, et al. 2016. Osteomyelitis caused by Veillonellaspecies: case report and review of the literature. J Infect Chemother 22:417-420.

7. Chen, Y.C., P. H. Ko, C. J. Yang, Y. C. Chen, C. J. Lay, C. C. Tsai, et al. 2016. Epidural abscess caused by Veillonella parvula : case report and review of the literature. J Microbiol Immunol Infect 49:804-808.

8. Marriott, D., D. Stark, and J. Harkness. 2007. Veillonella parvula discitis and secondary bacteremia: a rare infection complicating endoscopy and colonoscopy? J Clin Microbiol 45:672-674.

9. Isner-Horobeti, M. E., J. Lecocq, A. Dupeyron, S. J. De Martino, P. Froehlig, and P. Vautravers. 2006. Veillonella discitis. A case report. Joint Bone Spine 73:113-115.

10. Jean, M., J. O. Irisson, G. Gras, F. Bouchand, D. Simo, C. Duran, et al. 2017. Diagnostic delay of pyogenic vertebral osteomyelitis and its associated factors. Scand J Rheumatol 46:64-68.

Table 1 . Laboratory data of the patient

\begin{tabular}{lll}
\hline Variable & Reference range & Value on admission \\
\hline Red blood cell $($ per $\mu \mathrm{L})$ & $3860000-4920000$ & 4620000 \\
Hemoglobin $(\mathrm{g} / \mathrm{dL})$ & $11.6-14.8$ & 11.8 \\
Hematocrit $(\%)$ & $35.1-44.4$ & 37.7 \\
White blood cell (per $\mu \mathrm{L})$ & $3300-8600$ & 16200 \\
Platelets $($ per $\mu \mathrm{L})$ & $158000-348000$ & 1020000 \\
Sodium $(\mathrm{mmol} / \mathrm{L})$ & $135-145$ & 136 \\
Potassium $(\mathrm{mmol} / \mathrm{L})$ & $3.6-4.8$ & 8.0 \\
Chloride $(\mathrm{mmol} / \mathrm{L})$ & $101-108$ & 100
\end{tabular}




\begin{tabular}{lll}
\hline Variable & Reference range & Value on admission \\
\hline Urea nitrogen $(\mathrm{mg} / \mathrm{dL})$ & $8.0-20.0$ & 80.6 \\
Creatinine $(\mathrm{mg} / \mathrm{dL})$ & $0.46-0.79$ & 14.46 \\
Alanine aminotransferase (U/L) & $13-30$ & 86 \\
Aspartate aminotransferase (U/L) & $7-23$ & 21 \\
Alkaline phosphatase (U/L) & $106-322$ & 378 \\
C-reactive protein $(\mathrm{mg} / \mathrm{dL})$ & $0.0-0.1$ & 41.7 \\
\hline
\end{tabular}

Table 2. Antimicrobial susceptibility of this patient

\begin{tabular}{ll}
\hline Antimicrobial & Minimal inhibitory concentration $(\mu \mathrm{g} / \mathrm{mL})$ \\
\hline Ampicillin & {$[?] 0.5$} \\
Piperacillin & 8 \\
Cefaclor & {$[?] 8$} \\
Cefotiam & 16 \\
Cefotaxime & 16 \\
Ceftazidime & {$[?] 1$} \\
Ceftriaxone & 4 \\
Flomoxef & {$[?] 1$} \\
Azithromycin & 16 \\
Imipenem and cilastatin & 2 \\
Ampicillin and sulbactam & {$[?] 0.5$} \\
Piperacillin and tazobactam & 4 \\
Gentamycin & $>16$ \\
Clindamycin & $>4$ \\
Minocycline & {$[?] 2$} \\
Levofloxacin & {$[?] 0.5$} \\
\hline
\end{tabular}

Figure 1. 


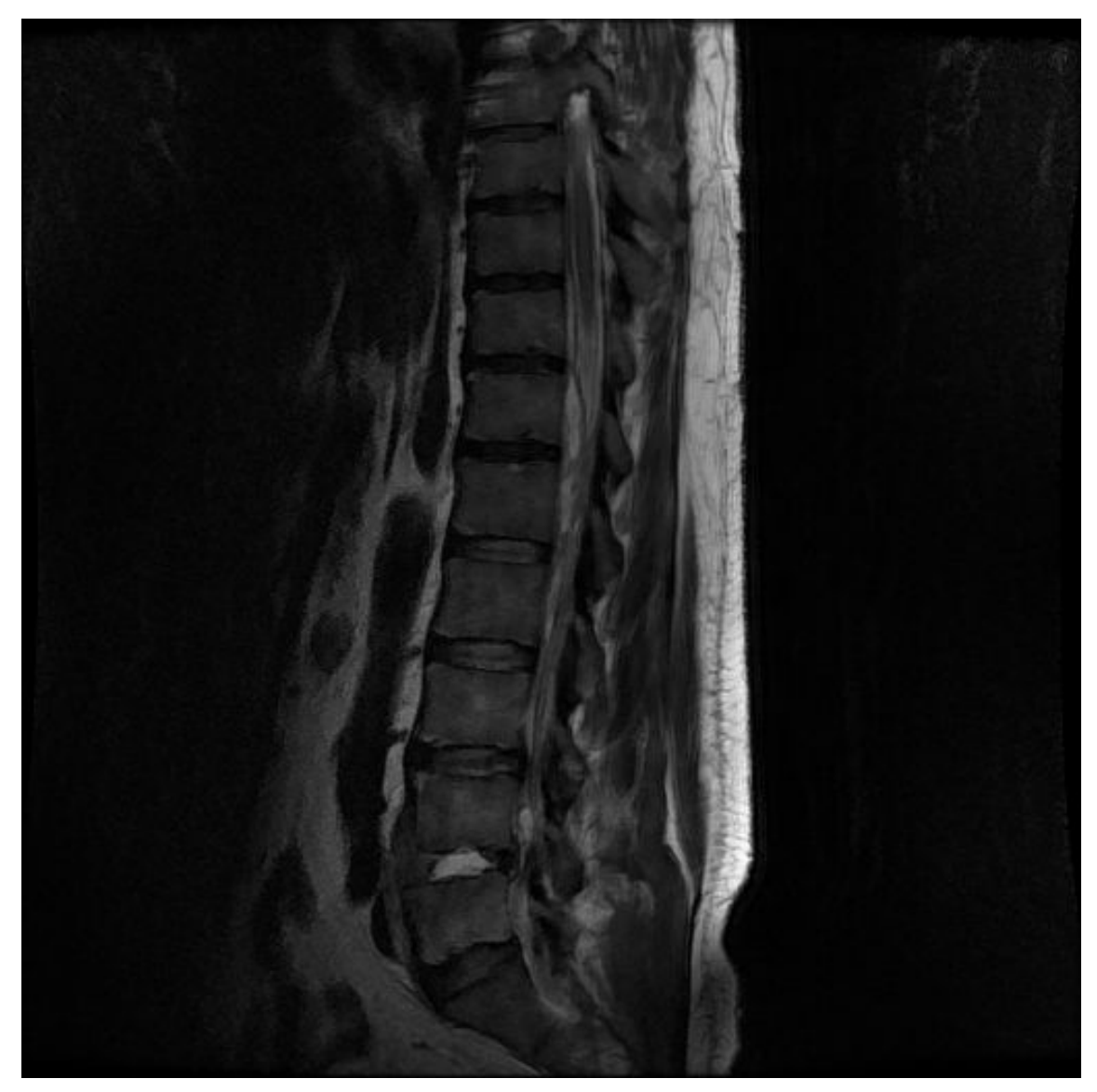

Figure Legend

Figure 1. Lumbar magnetic resonance imaging on the second day of hospitalization. The image shows an extensive epidural abscess with evidence of discitis in the L5/S1 region. 
